\title{
A Study on Features for Improving Performance of Chinese OCR by Machine Learning
}

\author{
Chul Kim \\ George Mason University, Korea \\ Incheon, Republic of Korea \\ ckim50@gmu.edu
}

\author{
Jang Su Kim, U Ju Kim \\ Pyongyang University of Science and Technology \\ Pyongyang, Democratic People’s Republic of Korea
}

\begin{abstract}
This paper discusses a method to improve the performance of Chinese OCR by choosing a proper feature vector and synthetic classification. We compare two groups of features which are used to implement Chinese OCR System and demonstrate that the first group of features is more useful for static Chinese OCR System. By now feature extractions have been done either for local features or for global features. Classifications have been done by single classification. We propose synthetic features extraction and classification in this paper. We find that the result is improved by machine learning method. Later we apply the result in the area of off- and on-line signature verification system.
\end{abstract}

\section{CCS CONCEPTS}

- Computing methodologies $\rightarrow$ Model verification and validation; • Software and its engineering $\rightarrow$ Abstraction, modeling and modularity.

\section{KEYWORDS}

OCR, feature selection, Chinese character recognition, pattern recognition, machine learning, authentication, biometrics

ACM Reference Format:

Chul Kim, Jang Su Kim, and U Ju Kim. 2019. A Study on Features for Improving Performance of Chinese OCR by Machine Learning. In 2019 The 3rd High Performance Computing and Cluster Technologies Conference (HPCCT 2019), June 22-24, 2019, Guangzhou, China. ACM, New York, NY, USA, 5 pages. https://doi.org/10.1145/3341069.3342991

\section{INTRODUCTION}

Printed Chinese Character Recognition (PCCR) systems have been widely used to automate the conversion into Chinese digital documents. One of the most challenging problem in Chinese OCR is that Chinese characters that are very close in shapes while their meanings are quite different [2]. Like other East-Asian languages, the structural components of Chinese characters are very complicated. What is more, even though two Chinese Characters have very similar structural elements, their meanings are quite different.

Permission to make digital or hard copies of all or part of this work for personal or classroom use is granted without fee provided that copies are not made or distributed for profit or commercial advantage and that copies bear this notice and the full citation on the first page. Copyrights for components of this work owned by others than ACM must be honored. Abstracting with credit is permitted. To copy otherwise, or republish, to post on servers or to redistribute to lists, requires prior specific permission and/or a fee. Request permissions from permissions@acm.org.

HPCCT 2019, June 22-24, 2019, Guangzhou, China

(C) 2019 Association for Computing Machinery.

ACM ISBN 978-1-4503-7185-8/19/06 . .\$15.00

https://doi.org/10.1145/3341069.3342991
The precision of an OCR system mainly depends on the features extracted to classify characters in the class set. Specifically, it depends on the number of extracted feature and how much complicated the processes of feature extractions are. The more useful features which have strong classification abilities, the better precision of OCR will be guaranteed. However, it will take longer time to classify a single Chinese Character. Therefore, it is crucial for improving the performance of the OCR engine to choose proper features which reflects the properties of characters. And the length of the feature vector affects the speed and precision: more prolonged feature vector increase the complexity and shorter feature vector drops the accuracy of the recognition. Simon Liao, Amy Chiang, and Miroslaw Pawlak introduced Gagenbauer moments [2]. Cheng-Lin Liu used shape normalization to extract robust features [3]. Wang Zhen-Yan used HMM for classification of Chinese Characters [4]. Liangbin Zheng, Ruqi Chen and Xiaojin Cheng selected BPN for classification [5]. He Zhiguo and Yang Xianli employed kernel independent component analysis [6]. Before this, Qiang Huo, Zhi-Dan Feng and Yong Ge tried to use Gabor features for Chinese OCR [7].

\section{FEATURE VECTORS AND CHARACTER CLASSIFIER}

\subsection{Feature Vector}

Optical feature of a character reflects the perceptual feeling by the morphological properties. The optical features are differentiated from fractal dimensions and wavelet features. It directly reflects the morphological property of the character. An example of the Chinese language, the optical property of a Chinese character is the ratio of the width and height, the complexity of the structure. Chinese character 我 has the following features.

- It consists of two parts.

- It is approximately a square and ratio of width and height is $1: 1$.

- The complexity of the character is medium.

- There is one horizontal stroke.

- There are two vertical strokes.

- There are two diagonal strokes.

- There are three dot-like strokes.

- Strokes are evenly spread over the rectangular area.

Additionally, it is worth pointing out that the relationships between the positions of the strokes are significant. A character image is normalized in the dimension of $48^{*} 48$ to extract features.

We suggest two groups of features. The first group of features consists of the followings:

- Ratio: 1 
- Hu-Moments: 3

- massive central coordinates: $2^{*}(9+1)=20$

- x-projection: 48

- y-projection: 48

- shape numbers: $4^{*}(9+1)=40$

- perimeter length: $2^{*}(9+1)=20$

Total number of features or length of feature vector is

$$
1+3+20+48+48+40+20=170 .
$$

The second group of features consists of the followings:

- Ratio: 1

- Hu-Moments: 3

- Gegenbauer Moments: 4

- Direction Contributivity: 4

- Shape Number: 4

- Directional Complexity: 4

- Perimeter Length: 1

- First and Second Zero-Cross: 24, 24

The total number of features or length of the feature vector is

$$
1+3+4+4+4+4+1+24+24=69 .
$$

We explain each kind of features in details below.

1. The ratio of width to the height of the wrapping rectangle

For each character, there exists a unique wrapping rectangle which has the minimum area. The ratio of width to height means those of this wrapping rectangle.

\section{Hu-Moment [1]}

A central moment is the same as the moments just described except that the mean values displace the values of $\mathrm{x}$ and $\mathrm{y}$ used in the formulas:

$$
\mu_{(p, q)}=\sum_{i=0}^{n} I(x, y)\left(x-x_{a v g}\right)^{p}\left(y-y_{a v g}\right)^{q}
$$

where

$$
x_{a v g}=\frac{m_{10}}{m_{00}} \quad \text { and } ; y_{a v g}=\frac{m_{01}}{m_{00}} .
$$

The central moment is invariant with the movement of the image

$$
\eta(p, q)=\frac{\mu_{p, q}}{m_{00}^{\frac{p+q}{2}+1}} .
$$

Finally, Finally, the Hu invariant moments are linear combinations of the central moments. The idea here is that, by combining the different normalized central moments, it is possible to create invariant functions representing different aspects of the image. It is invariant to scale, rotation, and (for all but the one called $h_{1}$ ) reflection.

$$
\begin{gathered}
h_{1}=\eta_{20}+\eta_{02}, h_{2}=\left(\eta_{20}-\eta_{02}\right)^{2}+4 \eta_{11}^{2}, \\
h_{3}=\left(\eta_{30}-3 \eta_{12}\right)^{2}+\left(3 \eta_{21}-\eta_{03}\right)^{2} .
\end{gathered}
$$

There are four more Hu-Moments $h_{4}, h_{5}, h_{6}, h_{7}$. The result of the calculation of Hu-Moments shows that $h_{1}$ to $h_{3}$ reflect the feature of the Chinese characters. But $h_{4}$ to $h_{7}$ do not correlate with the element of the Chinese characters. Depending on this result, we use the first three hu-moments as features.

\section{Coordinates of the Massive Central Points}

Usually, the central point's coordinates indicate which side the distribution of the character is biased. The result of the investigation of Chinese characters shows that the central points of the characters have enough discriminating ability. Therefore, $\mathrm{x}$ and $\mathrm{y}$ coordinates can be the features of a character. Additionally, we evenly divide a Chinese character into $3^{*} 3$ subparts. And the central points' coordinates of each part are additional features to strengthen the discriminating ability. Because even though the coordinates of the massive central points could be identical, it is impossible for subparts' central points to be identical.

4. $x, y$ - Projection [10]

$x, y$ - projection features are obtained by projecting the character image to $x$ - axis and $y$ - axis, respectively. This kind of features reflects the overall morphological properties.

5. Shape Numbers [8] The shape numbers are features which
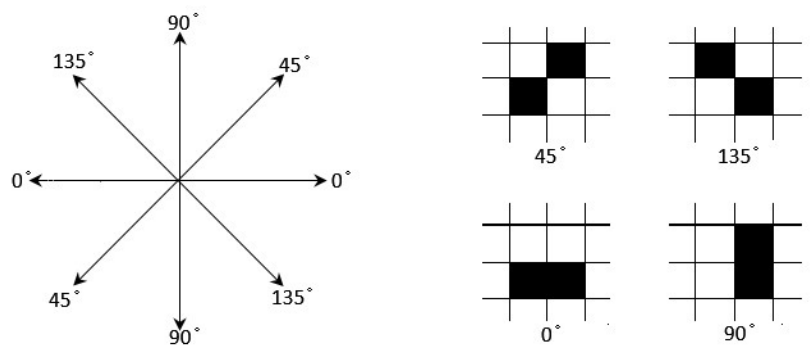

Figure 1: Directions of edge

reflects the perimeter shape of the image. A Freeman chain is a representation of a polygon in terms of a sequence of "moves". Each move is of a fixed length and in a particular direction. As can be seen in the illustration, there are eight cases of moving from one point to the next along the perimeter of a figure. The histogram of the eight numbers can be the feature of the character. In this paper, we suggest four instead of eight numbers. The reason is that $0^{\circ}$-direction is the same with $180^{\circ}$ - direction and $90^{\circ}$-direction are the same with $-90^{\circ}$-direction, and for the same reason, $45^{\circ}$-direction is same with $-135^{\circ}$-direction and $135^{\circ}$-direction is treated as the same with $-45^{\circ}$-direction because when iterating in clockwise and in anti-clockwise, these directions are interchangeable with each other.

6. Perimeter Length

The length of the perimeter of the character is also a feature. In conjunction with the other features, a Chinese character is divided into $3^{*} 3$ subparts and measure the boundaries of each subpart. These numbers are also featuring to maximize the discrimination between characters.

\section{Gegenbauer Moments}

The Gegenbauer polynomial is a class of orthogonal polynomials on the interval $[-1,1]$ characterized by a single parameter $\lambda$ that allows changing the form of the polynomial. Hence let $G_{n}(x ; \lambda)$ denote the Gegenbauer polynomial of order $n$ with the parameter $\lambda$, which can be any real number 
satisfying the restriction of $\lambda>-0.5$.

$$
G_{n}(x ; \lambda)=\frac{\Gamma\left(\lambda+\frac{1}{2}\right)}{\Gamma(2 \lambda)} \frac{\Gamma(n+2 \lambda)}{\Gamma\left(n+\lambda+\frac{1}{2}\right)} P_{n}^{\left(\lambda-\frac{1}{2}, \frac{\lambda-1}{2}\right)}(x),
$$

where $P_{n}^{(a, b)}(x)$ is Jacobi polynomial.

$$
A_{n}(\lambda)=c_{n}(\lambda) \int_{-1}^{1} f(t) G_{n}(x ; \lambda)\left(1-t^{2}\right)^{\lambda-\frac{1}{2}} d t
$$

With normalizing constant

$$
c_{n}(\lambda)=\frac{2^{2 \lambda} \Gamma^{2}(\lambda)}{2 \pi} \frac{n !}{\Gamma(n+2 \lambda)}(n+\lambda), \quad \lambda \neq 0
$$

We can define the $(n, m)$ Gegenbauer moments in a twodimensional space as follows:

$$
\begin{aligned}
& \left.A_{(} p, q\right)(\lambda) \\
= & c_{p}(\lambda) \int_{-1}^{1} \int_{-1}^{1} f(u, v) G_{p}(u ; \lambda) G_{q}(v ; \lambda) \\
& \left(1-u^{2}\right)^{\lambda-\frac{1}{2}}\left(1-v^{2}\right)^{\lambda-\frac{1}{2}} d u d v
\end{aligned}
$$

The following combinations are adopted as features:

$$
\begin{aligned}
& f_{1}=A_{2,0}(\lambda)+A_{0,2}(\lambda) \\
& f_{2}=\sqrt{\left(A_{2,0}(\lambda)-A_{0,2}(\lambda)\right)^{2}+4 A_{1,1}(\lambda)} \\
& f_{3}=\sqrt{\left(A_{3,0}(\lambda)-3 A_{1,2}(\lambda)\right)^{2}+\left(A_{0,3}(\lambda)-3 A_{2,1}(\lambda)\right)^{2}} \\
& f_{4}=A_{3,0}(\lambda)+A_{0,3}(\lambda)
\end{aligned}
$$

The Gegenbauer Moments are stable to rotation, scaling, and moving.

\section{Direction Contribution}

Direction contribution $L_{x}$ means how long the total of strokes in direction $x$ is.

$$
L_{x}=\text { sum of total pixels which have direction } x
$$

\section{Directional Complexity}

Directional complexity $C_{X}$ means that how complex a character is in the $x$-direction.

$$
C_{X}=\frac{L_{Y}}{\sigma_{X}}, \quad C_{Y}=\frac{L_{X}}{\sigma_{Y}}, \quad \sigma_{x}^{2}=\frac{\left.\sum(X-\bar{X})^{2}\right)}{n}, \quad \bar{X}=\frac{\sum \sum i C_{i j}}{n}
$$

where $L_{X}, L_{Y}$ mean directional contribution in $x, y$ directions.

\section{First and Second Zero-Cross}

First Zero-Cross in row $i$ means how many background pixels should pass to meet the first foreground pixel in row $i$ We can think of First Zero-Cross in columns as well. We explain the Second Zero-Cross in the same way.

These two groups of features have a good contrast: the first group is larger while the second one smaller. The first group take longer time to extract while the second one is shorter. And what's important is that, according to our experiments, the first group of features gives higher accuracy than the second group. In addition to that, the first group of features needs longer time to classify a character while the second one needs shorter.

We suggest that the first group of features is better than the second one because what we need is higher accuracy.

\subsection{Character Classifier}

A classification task begins with a data set in which the class assignments are known. The term Classifier is often used to refer to an algorithm that performs classification. It will be referred to Support
Vector Machine (SVM - Classification algorithm that uses a boundary to separate the data into two or more categories/classes) and k-Nearest-Neighbor (KNN - Classification algorithm that defines a data point's category as a function of the nearest $\mathrm{k}$ data points to it.) The trained data is the pairs of the feature vector and its class. Therefore, like common pattern recognition problems, in the case of character recognition, the distance between vector and vector, the distance between vector and class should be set correctly.

In the case of the Chinese language, the searching space is vast. It is almost impossible to satisfy the responsibility by using only one classification method. The number of Chinese characters is nearly 5000. If each character has 40 samples, the size of searching space is $40 * 5000=200,000$ in case of using KKNN in 170th dimension. Basic experiments show that KKNN is superior to SVM and NN in case of the Chinese language. But 200,000 times of comparing takes a long time.

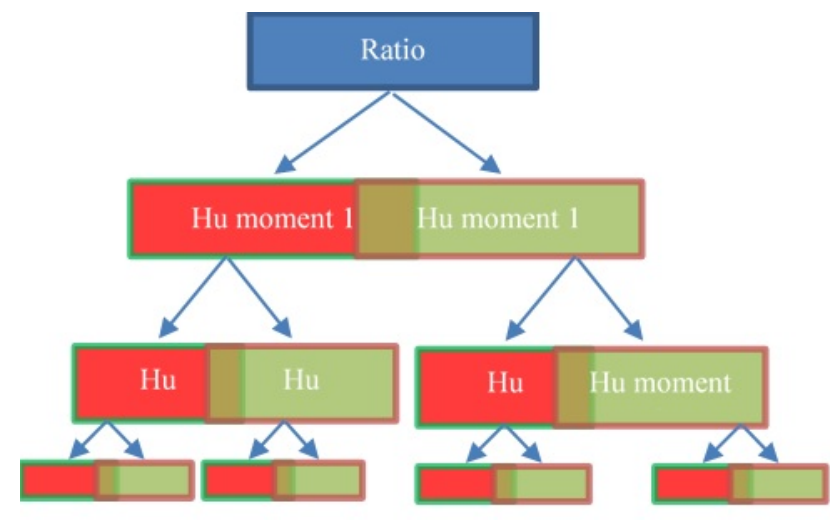

Figure 2: Binary classification tree

Therefore, this paper suggests synthetic classification by combining classifying tree and KKNN. In this case, we indicate the overlap between the child nodes to cope with the variety of shapes. What is essential is that which feature to select as nodes and how extensive the overlap should be. KKNN method employs Mahalanobis distance as the distance between vectors. Mahalanobis distance defines the distance between vectors as followings.

$$
\begin{array}{ll}
\bar{X} & =\left(x_{0}, x_{1}, x_{2}, \cdots x_{n}\right) \\
\bar{Y} & =\left(y_{0}, y_{1}, y_{2}, \cdots y_{n}\right) \\
\delta_{M d}(\bar{X}, \bar{Y}) & =(\bar{X}-\bar{Y})^{T} \Sigma^{-1}(\bar{X}-\bar{Y})
\end{array}
$$

Here $\sum$ is the covariance matrix of the trained data feature vector.

\subsection{Synthetic Classification}

According to our observation, those features suggested in this paper all function as a weak classifier. If we classically use all these features, the accuracy is dramatically high. The reason is that the linear combination of many weak classifiers works as a robust classifier. Using 170 features classically costs lousy performance. And using tree classifier decreases accuracy. So, combining these two methods can be a solution to provide speed and precision.

The synthetic method supposed in this paper first tries to reduce the searching space by using tree classifier. If it does not hit, 
Table 1: Comparison of two methods and Combination Result

\begin{tabular}{lccr}
\hline \multicolumn{1}{c}{ Binary Tree Classifier } & Classic Classifier & Synthetic Method \\
\hline accuracy & $\begin{array}{l}\text { 25\% for depth 3, 40\% for depth 2, 73\% for depth 1 } \\
\text { (Pivot features: hu-moment 1 for the first division, } \\
\text { x-axis massive central coordinate for the second division, } \\
\text { y-axis massive central coordinate for second division) }\end{array}$ & $92.4 \%$ \\
& 7ms for depth 3, 15ms for depth 2, 32ms for depth 1 & $98 \%$ & \\
(In Intel Core i3) & 92ms / character & 45ms / character \\
\hline
\end{tabular}

works the classical classification. To be detailed, we first search for a matching character in one of the leaf spaces of the binary classification tree, which is selected by the criteria of the feature values. If there is no good candidate character which satisfies the threshold of matching degree, we extend the searching space to the next leaf of the binary classification tree until we finally take the matching character. Or we conclude that the observed feature vector does not represent a Chinese character.

\section{DISCUSSION}

We select $156^{t h}, 157^{t h}, 169^{t h}$ features (Hu-Moment, $x$ - coordinate of the massive centre, $y$ - coordinate of massive centre). These features are the top three which have the best separability because, for these features, the in-class deviation is the minimum while the between-class difference is the maximum. But even these best ones do not have high separability.

There are few features which are suitable for separation among we suggest in this paper except these three. We extract proposed features and hu-moment $1, x$ - coordinate and $y$ - coordinate of the massive central point are selected as child nodes. $20 \%$ of the range is overlapped between child nodes. The number of child nodes is eight in the binary tree. KKNN is applied to the child node to evaluate the performance of the Desktop and Android Environment. 25\% in Table 1 means that the probability that we will find a matching character in the first searching space (leaf of the binary classification tree). It occurs we build a 3-depth binary tree with eight leaves. In the same way, we can find a matching character in the first searching space by $40 \%$ of probability in case of a two-depth binary tree with four leaves and by $73 \%$ in case of a one-depth binary tree with two leaves.

We set four criteria to distinguish groups of features. There are time measurements to extract and classify, accuracy and performance. These criteria provide meaningful separations of features. Table 2 shows the quantitative results of these four criteria. While we use the features together, we could reach high accuracy and efficiency.

For example, Figure 3 shows the Hu-Moment distribution, which used as the first pivot element.

As shown in the figure, we could obtain a distinguishable result for the inputs throughout the feature, and along with other features, the proposed system provides better outcomes for Chinese OCR system.

We implement 170 features in a system, and we get the following results of performance. We compare the effects on Java application and Android APK as shown in Table 3.
Table 2: Comparison performance and accuracy of two groups of features

\begin{tabular}{lcr}
\hline & $\begin{array}{c}\text { The first group } \\
\text { of features }\end{array}$ & $\begin{array}{r}\text { The second group } \\
\text { of features }\end{array}$ \\
\hline Time to extract & $60 \mathrm{~ms} /$ character & $10.5 \mathrm{~ms} /$ character \\
Time to classify & $32 \mathrm{~ms} /$ character & $16 \mathrm{~ms} /$ character \\
Accuracy & $98 \%$ & $75 \%$ \\
Performance & $92 \mathrm{~ms} /$ character & $26.5 \mathrm{~ms} /$ character \\
\hline
\end{tabular}

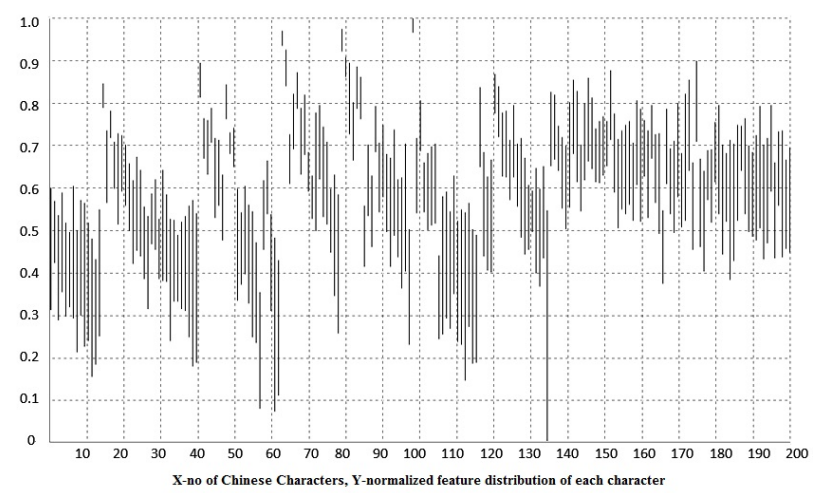

Figure 3: Hu-Moment distribution

\section{CONCLUSION}

This paper suggests 170 features of 5 classes which extracted to reflect the property of Chinese characters. Also classifying tree and KKNN are synthesized to improve the performance in the implemented system. When we implemented the features and experimented, we found that there is significant separability in- and inter-classes. Finally, we tested the system in two different environments and achieved validation and verification of the system.

For future work, we are going to implement and construct offand on-line signature verification system, which is one of the prominent methods for behavioural authentication.

\section{REFERENCES}

[1] Gary Bradski and Adrian Kaehler, "Learning OpenCV", OReilly, 2008, pp.253 256 
[2] Simon Liao and Amy Chiang and Qin Lu and Miroslaw Pawlak, "Chinese Character Recognition via Gegenbauer Moments", 2011

[3] Cheng-Lin Liu, "Handwritten Chinese Character recognition: Effects of shape normalization and features extraction", IEEE Conference Publication, 2017

[4] Wang Zhen-Yan, "Chinese Character Recognition Method Based on Image Processing and Hidden Markov Model”, Intelligent Systems Design and Engineering Applications (ISDEA), 2014 Fifth International Conference

[5] Liangbin Zheng and Ruqi Chen and Xiaojin Cheng, "Research on offline Handwritten Chinese Character Recognition Based on BP Neural Networks", 2011 International Conference on Computer Science and Information Technology

[6] He Zhiguo and Yang Xiaoli, "Resarch on Feature Extraction Method for Handwritten Chinese Character Recognition Based on Kernel Independent Component Analysis”, Research Journal of Applied Science, Engineering and Technology, 2013

[7] Qiang Huo and Zhi-Dan Feng and Yong Ge, "A Study on the use of Gabor features for Chinese OCR”, Proceedings of 2001 International Symposium of Intelligent Multimedia, Video and Speech Processing

[8] Wing Hang Cheung, "Chinese Optical Character Recognition for Information Extraction from Video Images”, 2012

[9] An-Bang Wang and Kuo-Chin Fan, "Optical Character Recognition of Handwritten Chinese Characters by Hierarchical radical matching method", 2014

[10] Andreas Hanemann, "Optical Character Recognition of Chinese Characters", 2003

Table 3: Comparison performance and accuracy of two groups of features

\begin{tabular}{ccc}
\hline & Java Application & Android APK \\
\hline Physical Environment & Intel Core i3 2.53GHz RAM 4GB & Pyongyang-2402-S700 CPU 1GHz RAM 1GB \\
Runtime Environment & Window 7 + JDK8.0 & Android 4.4 \\
Accuracy & $92.4 \%$ & $92.2 \%$ \\
Speed of Recognition per Character & $0.05 \mathrm{~s}$ & $0.4 \mathrm{~s}$ \\
Number of Trained Chinese Characters & 4952 & 4952 \\
Number of Samples in Trained Data & 40 & 40 \\
Number of Features & 170 & 170 \\
Character Segmentation Precision & $99.5 \%$ & $99.4 \%$ \\
\hline
\end{tabular}

